\title{
The Effects of Shock Strength on Droplet Breakup
}

\author{
Jomela C. Meng and Tim Colonius
}

\section{Introduction}

The breakup of droplets occurs in the combustion of multiphase mixtures and the atomization of liquid jets. Most experiments studying droplet breakup have used the passage of a normal shock to provide a step change to uniform flow conditions. In the late 1950s, Engel [2] investigated how water droplets of various sizes broke up in the flow behind air shocks of different strengths. The subsequent deformation and breakup of the water droplets were captured using spark pictures, and a fine mist was observed to develop in the wake of the disintegrating droplet. Using drift measurements, an attempt was made to calculate the accelerations and unsteady drag coefficients of the deforming water droplets. Engel's analysis concluded that the drag coefficient compared well to that for a perforated disk. In his recent review, Theofanous [10] summarized the aerobreakup of both Newtonian and viscoelastic liquid drops, and highlighted the criticalities in the breakup process. Also discussed were the interactions between the Kelvin-Helmholtz and Rayleigh-Taylor instabilities, and the interplay of the multitude of length and time scales present in the problem. Using the novel diagnostic of laser-induced fluorescence, the experiments of Theofanous et al. [11] were able to achieve the necessary temporal and spatial resolutions to identify two primary breakup regimes: Rayleigh-Taylor piercing and shear-induced entrainment.

Unfortunately, three-dimensional (3D) simulations are often too computationally expensive to allow direct comparisons with droplet experiments. Therefore, breakup is often studied using two-dimensional (2D) simulations which are physically equivalent to the breakup of liquid columns or cylinders. The work of Igra and Takayama $[4,5]$ provides useful validation for $2 \mathrm{D}$ simulations since their experiments investigated the breakup of water cylinders in the flow behind a shock wave. Columns with initial diameters of 4.8 and $6.4 \mathrm{~mm}$ were impacted by Mach 1.18, 1.30, 1.47,

California Institute of Technology

1200 E. California Blvd.,Pasadena, CA 91125 U.S.A. 
and 1.73 shocks in air. In the present work, the experiments of Igra and Takayama $[4,5]$ are first duplicated for validation purposes. Then, by extending the range of simulated shock strengths, the effects of the transition from subsonic to supersonic freestream flow around the water cylinder are investigated.

\section{Model and Methodology}

The breakup of a water cylinder is simulated on a rectangular Cartesian grid comprised of 1200x600 cells. The grid has a nominal resolution of 100 cells per original cylinder diameter, and grid stretching is employed in the streamwise direction as the boundaries are approached. Only half of the water cylinder is simulated and a symmetric boundary condition is enforced along the bottom of the domain. Non-reflecting boundary conditions are applied everywhere else. The water cylinder and the air downstream of the planar shock are initially at rest. At the start of the simulation, the shock is set in motion towards the water cylinder and establishes a steady freestream flow field. The range of simulated shock strengths and their corresponding post-shock Mach numbers are shown in Table 1. Within the literature,

Table 1 Post-shock Mach numbers in the shock-stationary (SS) and shock-moving (SM) reference frames.

\begin{tabular}{lcccccc}
\hline$M_{S}$ & 1.18 & 1.30 & 1.47 & 1.73 & 2.00 & 2.50 \\
\hline$M_{2}(S S)$ & 0.8549 & 0.7860 & 0.7120 & 0.6330 & 0.5774 & 0.5130 \\
$M_{2}(S M)$ & 0.2625 & 0.4056 & 0.5775 & 0.7885 & 0.9622 & 1.1970 \\
\hline
\end{tabular}

the Weber and Reynolds numbers have, respectively, been used to characterize the relative importance of inertial-to-capillary forces and inertial-to-viscous forces. Using the experimental parameters for a water cylinder in air, the approximate Weber numbers corresponding to the four weaker shock strengths range from 940-19,300. The Reynolds numbers corresponding to the same shock Mach numbers range from 39,900-237,600. These high Weber and Reynolds numbers suggest that the physical mechanisms of breakup are primarily driven by inertia, and that a reasonable first approximation can be made by neglecting the effects of surface tension and viscosity.

The flow is then governed by the multicomponent, compressible Euler equations. Following the five-equation model of Allaire et al. [1], the governing equations consist of a mass equation for each fluid, one mixture momentum equation, one mixture energy equation, and an advection equation for one of the volume fractions. Each fluid is considered to be inviscid, immiscible, and compressible. The stiffened gas equation of state [3] is used to close the system of equations, and models both gases and liquids in the flow solver. Material interfaces, in the absence of mass transfer and surface tension, are simply advected by the local velocity field, and are modeled using volume fractions. In the limit of infinite resolution, the interfaces are sharp, 
but shock- and interface-capturing schemes diffuse the interface. In this case, the nominal interface location is defined using a threshold liquid volume fraction above which the individual cell is considered to be part of the coherent water column. The mixture properties for cells in the transition regions are computed according to the mixture relations found in [1].

The numerical method is based on a finite-volume framework, and was shown by Johnsen and Colonius [6] to be both shock- and interface-capturing. Spatial reconstruction is accomplished with a third-order WENO scheme coupled with the HLLC Riemann solver. An explicit third-order total variation diminishing RungeKutta scheme is used to march the equations forward in time. The time step is chosen to limit the $\mathrm{CFL} \approx 0.25$ for stability. Time is non-dimensionalized by a characteristic breakup time, $t^{*}=t \frac{u_{0}}{d_{0}}$, found in the literature $[7,8,9]$.

Analysis of the cylinder's behavior requires computing the position, velocity, and acceleration of the cylinder's center of mass (COM). In addition to the straightforward calculation of the COM location, analytical expressions can be derived for the COM's velocity and acceleration, provided there is no flux of liquid mass across the domain boundaries.

$$
\overline{\mathbf{u}}=\frac{\int \alpha_{L} \rho_{L} \mathbf{u} d A}{\int \alpha_{L} \rho_{L} d A} ; \quad \overline{\mathbf{a}}=\frac{\int \alpha_{L} \rho_{L} \mathbf{a} d A}{\int \alpha_{L} \rho_{L} d A}
$$

where $\mathbf{u}$ and $\mathbf{a}$ are the local velocity and acceleration fields. (1) is easily computed by a discrete integration over the entire computational domain. Once liquid mass is lost through the boundaries, (1) is no longer valid, and the analysis is terminated.

\section{Results and Discussion}

To validate the computational results, comparison is first made with the experimental data in $[4,5]$. In order to quantify the time history of the cylinders' deformation, Igra and Takayama measured the centerline width and coherent body area from holographic interferograms. Since it was not possible to positively determine the criteria used to define the cylinder boundaries in $[4,5]$, an appropriate range of threshold volume fractions (shown in Fig. 1) is chosen in an attempt to bound the experimental data. Numerical measurements of the cylinders' centerline width and coherent body area compare well to the experimental data. Both the streamwise dimension of the cylinder and its coherent body area monotically decrease as the cylinder is compressed and material is stripped away from the cylinder's periphery. This mode of breakup is consistent with the features of stripping breakup expected for the relevant Weber numbers. From their visualizations, Igra and Takayama were able to track the drift of the water cylinders by measuring the location of the front of the cylinder. Figure 2 shows good agreement between simulation and experiment for the weaker shock strengths. For reasons not yet fully understood, the comparison deteriorates for the higher shock strengths. 

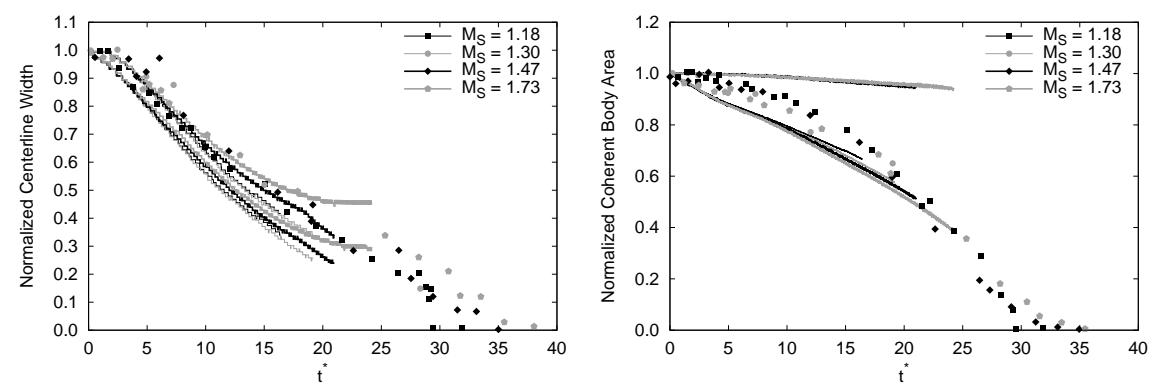

Fig. 1 Measurements of cylinder deformation. Discrete data represent experimental measurements. The two lines for each shock strength represent choosing threshold volume fractions of 0.50 and 0.99 . a centerline width. $\mathbf{b}$ coherent body area

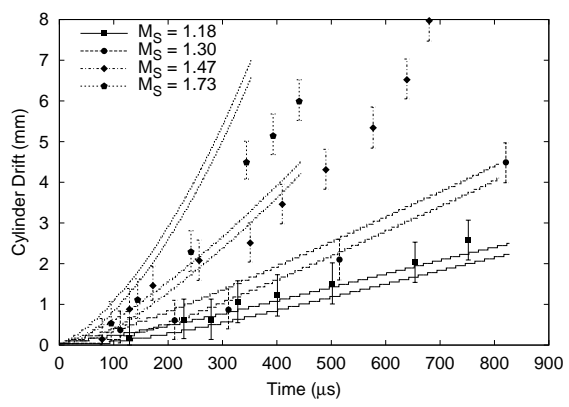

Fig. 2 Drift of the water cylinders as measured off the forward stagnation point.

Numerical schlieren images of the breakup of the water cylinders behind Mach 1.47 and 2.50 shocks are shown in Fig. 3. The supersonic velocity behind the Mach

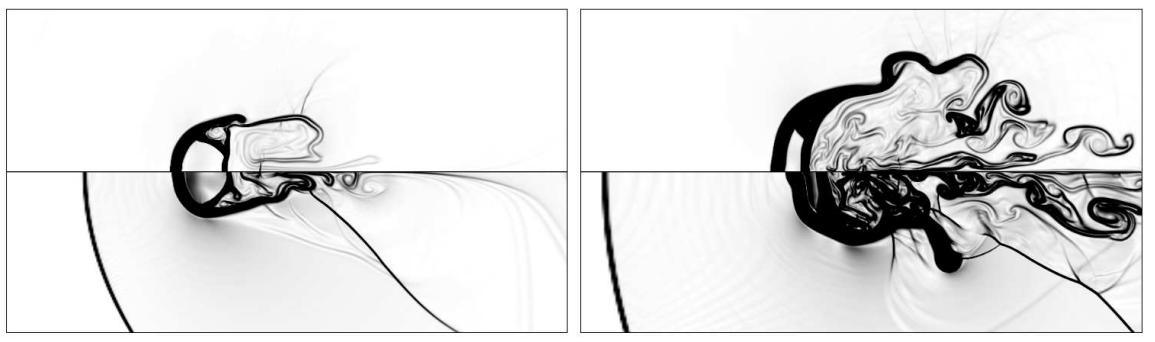

Fig. 3 Numerical schlieren images of the breakup behind Mach 1.47 (top) and Mach 2.50 (bottom) shockwaves at $\mathbf{a} t^{*}=6.213 \mathbf{b} t^{*}=15.533$

2.50 shock is evidenced by the detached bow shock preceding the deforming water cylinder. Cylinder wakes for supersonic post-shock flow appear to be narrower than their subsonic counterparts and standing shocks are visible along the length of the 
wake. Looking at the full range of simulated shocks, the drift and velocities of the cylinders' COM are shown in Fig. 4. The transition from subsonic to supersonic
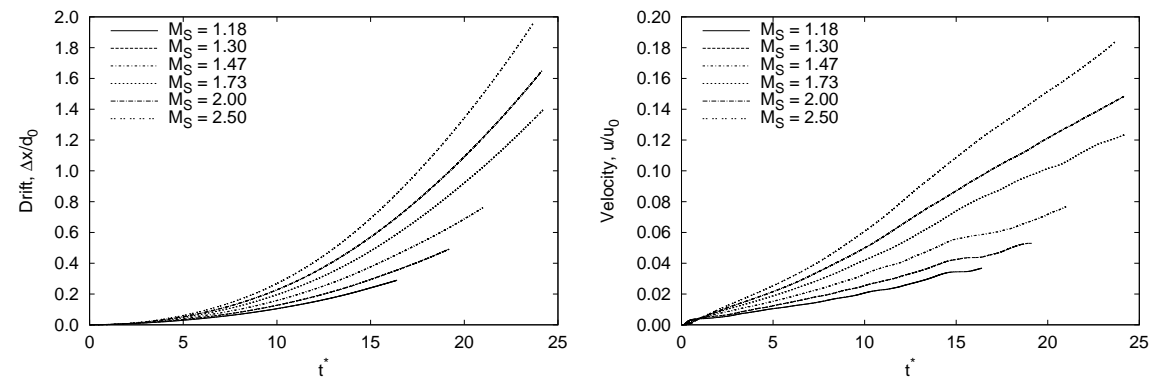

Fig. 4 a Drift of the cylinders' center of mass. b Velocity of the cylinders' center of mass

freestream flow speeds does not appear to significantly alter the cylinder's behavior. As the incident shock Mach number is increased, the faster post-shock flow around the cylinder induces greater cylinder velocities. The time history of the cylinder's trajectory remains a smooth, parabolic curve, which has led previous work to assume constant acceleration. However, fluctuations are observable in the velocity curves. The acceleration of the COM (as computed from the analytical expression in (1)) is shown as the left half of Fig. 5. Here, the acceleration has been non-dimensionalized as $a^{*}=a \frac{d_{0}}{u_{0}^{2}} \frac{p_{1}}{p_{2}}$. Using this scaling, the transient acceleration behavior collapses for all subsonic and supersonic post-shock velocities. Knowing all the relevant flow
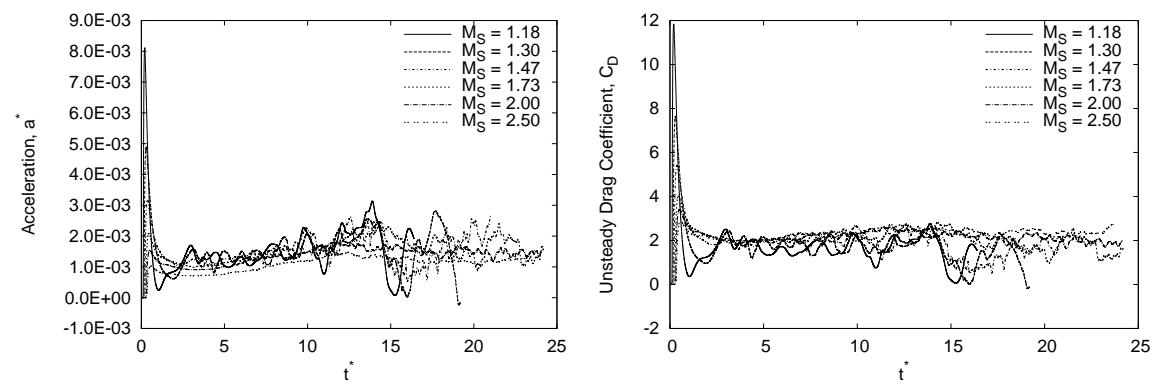

Fig. 5 a Acceleration of the cylinders' center of mass. b Unsteady drag coefficient of the deforming cylinders

variables, the unsteady drag coefficient can be calculated for the deforming water cylinder. The drag coefficient is defined as

$$
C_{D}=\frac{D}{\frac{1}{2} \rho u^{2} S}=\frac{m a}{\frac{1}{2} \rho_{G}\left(u_{0}-u\right)^{2} d}
$$


Using (2), the unsteady drag coefficients for different shock strengths collapse for a significant period of time. Though oscillations are present, they appear to fluctuate around a relatively steady value. It is notable that wave drag does not significantly alter the drag coefficient (as expected in the rigid body case). Since the water cylinder is undergoing large deformations, it would appear that parasitic drag is dominant.

\section{Conclusions}

In summary, the breakup of water cylinders was simulated and compared to experimental data. Measurements of the cylinders' drift and various benchmarks of deformation showed reasonable agreement between simulations and experiments. The transition from subsonic to supersonic post-shock flow speeds did not alter the similarity of the solutions, and collapse was shown for the unsteady acceleration and drag coefficient for all simulated shock strengths. When computed using the deformed diameter, the drag coefficient fluctuated around a relatively steady mean value. Future 3D simulations will capture the effects of the third dimensionality and facilitate comparisons with droplet experiments.

\section{References}

1. G. Allaire, S. Clerc, and S. Kokh. A five-equation model for the simulation of interfaces between compressible fluids. J. Comput. Phys., 181:577-616, 2002.

2. O. G. Engel. Fragmentation of waterdrops in the zone behind an air shock. J. Res. Nat. Bur. Stand., 60(3):245-280, March 1958.

3. F. H. Harlow and A. A. Amsden. Fluid dynamics. Technical Report LA-4700, LASL, June 1971.

4. D. Igra and K. Takayama. A study of shock wave loading on a cylindrical water column. Technical Report Vol. 13, pp. 19-36, Institute of Fluid Science, Tohoku University, March 2001.

5. D. Igra and K. Takayama. Experimental investigation of two cylindrical water columns subjected to planar shock wave loading. J. Fluid Eng. - T. ASME, 125:325-331, 2003.

6. E. Johnsen and T. Colonius. Implementation of WENO schemes in compressible multicomponent flow problems. J. Comput. Phys., 219:715-732, December 2006.

7. M. Pilch and C. A. Erdman. Use of breakup time data and velocity history data to predict the maximum size of stable fragments for acceleration-induced breakup of a liquid drop. Int. J. Multiphase Flow, 13(6):741-757, 1987.

8. A. A. Ranger and J. A. Nicholls. Aerodynamic shattering of liquid drops. AIAA J., (68-83), 1968.

9. P. G. Simpkins and E. L. Bales. Water-drop response to sudden accelerations. J. Fluid Mech., 55:629-639, 1972.

10. T. G. Theofanous. Aerobreakup of Newtonian and viscoelastic liquids. Annu. Rev. Fluid Mech., 43:661-690, 2011.

11. T. G. Theofanous, G. J. Li, and T. N. Dinh. Aerobreakup in rarefied supersonic gas flows. $J$. Fluid Eng. - T. ASME, 126:516-527, 2004. 